\title{
Why complex surgeries in children are associated with poorer outcomes
}

\author{
Mathias Johansen ${ }^{1, \star}$, Vincent Collard ${ }^{1}$, Thomas Engelhardt ${ }^{1}$
}

${ }^{1}$ Department of Anesthesia, McGill University Health Center, Montreal Children's Hospital, Montreal, QC, Canada

\section{*Correspondence}

mathias.fuglsang.johansen@regionh.dk (Mathias Johansen)

\begin{abstract}
Despite obvious progress in perioperative morbidity and mortality we still face challenges in explaining why complex surgical procedures in children are associated with poorer outcomes. This review aims to address the importance of improving perioperative outcome through the implementation of strategies for quality assurance and the safe conduct of paediatric anaesthesia.
\end{abstract}

\section{Keywords}

Complex surgery; Paediatric; Anaesthesia; Mortality; Morbidity; Caseload; Quality; Education; Neurotoxicity; Safetots

\section{Introduction}

The angst of administering potentially neurotoxic anaesthetic agents to young children has been a dominating research theme for the past 2 decades in pediatric anesthesia. This was further fuelled by a statement from the U.S. Food and Drug Administration in 2016 warning against the use of anesthetic agents in young children [1]. However, publication of several large clinical trials such as General Anesthesia Spinal (GAS), Pediatric Anesthesia Neurodevelopment Assessment (PANDA) and Mayo Anesthesia Safety in Kids (MASK) have provided strong evidence that a short exposure to general anesthesia for elective surgery at a young age does not result in relevant changes in neurodevelopmental outcome [2]. It is a long established fact that children undergoing anesthesia have an increased perioperative risk for morbidity and mortality when compared with adults [3]. Unique social, physiological and biological differences are now emerging as a renewed research focus leading to improvements in perioperative care in dedicated paediatric centers [4].

\section{Background}

Retrospective cohort studies suggest a link between exposure to anaesthesia/surgery at a young age and a subsequently altered neurobehavioral outcome [5]. This association combined with plausible biological data from laboratory research data demonstrating a direct neurotoxic effect of anaesthesia agents on the rodent brain, have resulted in a warning against the use of anesthetic agents for more than 3 hours in young children despite no direct clinical evidence [1, 6, 7]. It appears impossible to detangle the pharmacodynamic effects of the anaesthetic drugs from the multiple other perioperative factors as well as the underlying disease process. It appears prudent to acknowledge that it may never be possible to demonstrate these laboratory findings in conventional clinical trials. However, addressing the known risks of the increased morbidity and mortality in children remains the responsibility of the clinician and institution alike. A poor neurological outcome, prolonged cardio-respiratory depression and perioperative cardiac arrest in paediatric anaesthesia are directly linked to the (in) experience of the anaesthesiologist in charge $[8,9]$. In addition, this paper highlights why complex surgery and subsequently prolonged procedures in children are associated with poorer outcomes.

\section{Complex surgery}

There is no universal definition of complex surgery. Intrinsic (patient status and comorbidity) as well as extrinsic factors (organisational setup, educated staff and clinical experience) contribute to the overall perception of complexity impacting patients incidence of morbidity and mortality (Table 1). It appears obvious that children with associated co-morbidities, even with optimal care, are likely to have worse outcomes than their counterparts without health concerns. This has in part been emphasized by pediatric co-morbidity prediction models showing a strong correlation between the extent of co-morbidity and an increased in the 1-year mortality rate (Table 2). Although studied extensively in adults, the impact of co-morbidity has to date received little attention in children. The lack of scientific evidence and research in the field of anaesthesia impact on perioperative outcome makes it difficult to establish validated risk stratification models. Hence, the paediatric anaesthesia community must rely on maintenance of physiological homeostasis as the key for the safe conduct of anesthesia in children (Table 3).

During the past decades increased paediatric perioperative care has resulted in an overall mortality reduction. This has led clinicians and researchers to prioritise their focus of atten- 
TA B L E 1. Common Complex Surgical Procedures and Related Outcomes.

$\begin{array}{lcccccc}\text { Surgery } & \text { Mortality* } & \text { Complications/Morbidity* } & \text { Length of surgery** Length of Stay } & \text { References } \\ \text { Craniosynostosis } & 0.2 \% & 6.4-16.5 \% & 140-250 \text { minutes } & 1.6-3.7 & {[10,11]} \\ \text { Esophageal Atresia } & 7.5-9 \% & 62 \% & 200-600 \text { minutes*** } & 18-66 & {[12,13]} \\ \text { Congenital Diaphragmatic Hernia } & 21.1 \% & 12-77 \% & 110-200 \text { minutes } & 16-51 & {[14-16]} \\ \text { Congenital Cardiac Surgery } & 8.2 \% & 21.8 \% & 175-650 \text { minutes**** } & 7-10.9 & {[17-20]}\end{array}$

Mortality, morbidity, length of anaesthesia and length of stay are considered highly variable depending on (e.g.) pre-operative patient assessment, comorbidity, surgical approach and organizational setup. Length of surgery does not include preparation and waking up as well as need for postoperative sedation in the intensive care unit. (* Incidences of 30-day mortality and morbidity. Numbers extracted from a PubMed search are highly variable and not a valid estimate for long term survival. ** Length of surgery extracted from operating schedules at the Montreal Children's Hospital (Quebec, Canada) during a 5-year period. Timeframe does not include 'anaesthesia time' (preparation of patient, emergence from anaesthesia and possible need for continuous sedation in intensive care unit)).

*** The large variation is primarily due to multiple co-interventions during esophageal atresia repair. **** The large variation is dependent on the extent and severity of the surgical repair.

TA B L E 2. Impact of pediatric patient comorbidity on 1-year survival among children age 1-14 years. (adapted from Derek Tai et al. Development of Pediatric Comorbidity Prediction Model Arch Pediatr Adolesc Med. 2006 [21]). OR (odds ratio of dying); CI (95\% confidence interval).

\begin{tabular}{lc} 
Co-morbidity & OR $(95 \% \mathrm{CI})$ \\
\hline Brain cancer & $76.38(53.40-109.27)$ \\
\hline Diabetes insipidus & $39.23(20.75-74.17)$ \\
\hline Asphyxia & $25.49(19.74-32.91)$ \\
Shock & $23.69(14.61-38.42)$ \\
\hline Leukemia & $21.34(13.77-33.065)$ \\
Heart failure & $20.89(12.92-33.78)$ \\
\hline Feeding problem & $8.67(5.94-12.66)$ \\
\hline
\end{tabular}

tion towards ameliorating perioperative outcome in terms of morbidity and in particular neurodevelopmental delay. Surgical procedures in the young paediatric population of more than 3 hours are relatively rare and often related to children with pre-existing multiple organ failure which is known to play an important role in neurodevelopmental delay. Each year $2-3 \%$ of all neonates undergo anaesthesia and surgical procedures for non-cardiac congenital abnormalities (NCCA) [22]. A population which already at baseline has been found to possess several possible risk factors (preterm birth, low birth weight, chromosomal disorders, syndromes, length of hospital stay and duration of mechanical ventilation) predisposing neurocognitive behavioural disorders. Adding to this fact, the risk of poor perioperative anaesthetic management (Table 3), morbidity and long term survival will inevitably be affected. Prolonged and complex surgical procedures in patients with multiple comorbidities and risk factors constitute an increased perioperative risk of adverse events which is not solely limited to the decision making that takes place in the operating and recovery room [23]. The short- and long term outcomes should be considered a result of correct and timely primary diagnosis, preoperative optimization, perioperative maintenance of physiological homeostasis, optimal postoperative pain relief and psychosocial support of patients and family. An important task which is to be carried out and supported not only by clinicians but also key opinion leaders and administrators in the health care industry.

\section{Addressing competence}

Established in 2014, the SAFETOTs initiative has provided guidance of markers of quality in paediatric anaesthesia [1]. Demanding attention from regional and national societies to develop and implement strategies for quality improvement in paediatric anaesthesia, this initiative has shed light to one of the most obvious and yet clinical and scientifically understated reasons for a poor perioperative outcome in young children the "Who", "Where", "What", "When" and "How".

\subsection{Who should anaesthetize our youngest patients?}

The concept that every anaesthetist can proficiently look after all patients of all ages and co-morbidities are outdated. It is well known that anaesthetic management of children in inexperienced hands ( $<100$ cases per year) equals a substantial higher morbidity rate compared to experienced operators with a caseload of $>200$ per year [24]. A minimum annual caseload (including $1 \mathrm{infant} /$ per month/per anesthesiologist) is recommended to minimize complications (National Confidential Enquiries into Perioperative Deaths - NCEPOD) [25]. This is ideally achieved by national and regional societies to clinically and politically acknowledge and promote the importance of continuous medical education and centralization of high risk patient populations. This will enable the concentration of highrisk populations in specialized centers hence facilitating state of the art paediatric anaesthesia, a continuous maintenance of skills and $1: 1$ consultant/fellow-resident supervision for achieving the best academic up-to-date education [26]. To date this has become mandatory in some European countries. In Denmark, anesthesia in children younger than 2 years of age is no longer part of the anesthesiological curriculum and must be anaesthetized in special centres. In France, children less than 3 years of age have to be anesthetized by specialized pediatric 
TA B L E 3. Anaesthesia factors affecting perioperative morbidity and mortality. Table showing some of the most common anaesthesia related factors influencing perioperative morbidity and mortality [The 10-Ns Maintenance of physiological homeostasis is key for the Safe Conduct of Anesthesia in Children; www. safetots.org/quality-10n/l.

$\begin{array}{lll}\text { Homeostasis } & \text { Diagnosis } & \text { Comment } \\ \text { Normoxemia } & \text { Hypoxemia } & \text { May lead to adverse short (cardiac arrest) and long-term (brain ischemia) outcomes }\end{array}$

Normovolemia Hypovolemia Results in hypoperfusion and tissue hypoxia. The leading cause of perioperative cardiac arrests

Normotension Hypotension Deviations can lead to inadequate organ perfusion and damage

Normoglycemia Hypoglycemia Significant impact on cellular function and body metabolism (e.g. seizures and death)

Normocapnia Hypocapnia Changes in arterial carbon dioxide tensions significantly affect acid-base status, sympathomimetic tone and blood flow to vital organs (e.g. heart and brain)

Normonatremia Hyponatremia Acute perioperative changes in plasma sodium concentrations are a leading cause of avoidable morbidity and mortality in children

Normothermia Hypothermia Hypothermia during anesthesia has many harmful consequences and may trigger multiple physiological changes such as increased metabolic rate and oxygen consumption, prolonged bleeding time and increased risk for postoperative infection

anesthesiologists $[1,27,28]$. To date the Scandinavian Society of Anaesthesiology and Intensive care medicine (SSAI) has one of the most renowned and elaborate European specialist training programs in paediatric anaesthesia (https://ssai. info/education/paediatric-anaesthesia/) [1].

\subsection{Where should our youngest patients be treated to obtain best possible outcome?}

As in many other subspecialties the idea of pooling a high risk patient population to specialized centers has become more evident. The presence of paediatric centers facilitates a 24/7 paediatric anaesthesia service ensuring safety and continuity not only for induction and emergence of anaesthesia but also in terms of safe post-operative observation, pain service availability, specialized nursing rehabilitation and appropriate equipment. The establishment of such a structured pediatric anesthesia service involving the systematically implementation of departmental teaching, workshops, regulations and standard operating protocols (SOPs) must be considered gold standard. Hospitals responsible for elective loco-regional paediatric surgical service (private and public) should strongly consider relying on a joint collaboration with paediatric centers for obtaining sufficiently trained personnel.

\subsection{What to tell the parents, surgeons and referring doctors?}

To date there is no proven causal link between anesthetic agents and cerebral damage in neonates, infants and small children. However, there is a direct correlation between "poor anaesthetic management"/"lack of proper perioperative care" and development of long term neurological deficits and increased risk of mortality in small children. Hence, we should continuously emphasize the importance of the "Who" and "Where".

\subsection{When should surgery be performed?}

Optimal timing of surgery in children is crucial. Paediatric physiology is often characterised by showing only subtle signs of distress before a rapid deterioration occurs which might indicate a 'rather sooner than later' response. On the other hand it is well known that performing anaesthesia for medical and surgical procedures carries a greater risk of adverse events when performed outside normal working hours. Finally, performing anaesthesia for especially neonates and small children this should always be considered a last resort option. No evidence currently supports the idea of anaesthetic agents inducing neurotoxicity but this does not mean that we should carelessly induce, prolong or even expose our youngest patients to anaesthetic agents unless it is deemed absolutely necessary.

\subsection{How do we achieve the best possible outcome?}

However obvious it might seem, one of the greatest challenges in today's paediatric anaesthesia community is to align national and regional societies on best possible practice. For many years paediatric research has not been considered 'high profiled' and representing only a very small fraction of the entire health care industry this has resulted in a severe lack of financial support. A recently published European multicenter study (APRICOT) [29] demonstrated a great variability of how children are anaesthetized throughout Europe, resulting in a higher than expected incidence of critical events. Some of the answers to this problematic situation lie in the development and implementation of Standard Operating Protocols (SOPs). Based on the best available scientific evidence SOPs must define and describe not only what needs to be done, but also who is qualified to carry it out, and under what conditions the procedure can reliably be performed. In order to achieve this goal the paediatric anaesthesia community clearly needs to obtain consensus on finite outcome measures - in short - we need to speak the same language. A tremendous challenge and extensive work which is currently being investigated [30].

SAFETOTS $10-N$ is an initiative that in its simplicity guides clinicians to overall markers of safe paediatric anaesthesia conduct. We should emphasize the importance of maintaining perioperative homeostasis (normovolaemia, normotension, 
normoglycemia, normal heart rate, normoxemia, normocarbia, normal electrolytes, normothermia, no awareness and no pain) in order to avoid severe adverse events such as subclinical cerebral damage as a result of perioperative cardiac arrest, prolonged cardio-respiratory depression or cerebral edema which are all frequently results of a poor anesthesia management. While most of the 10-N outcome measures are rarely a problem for short term elective anaesthetic procedures in the older paediatric population it should be considered a very important key factor in providing anaesthesia and postoperative care (recovery room and intensive care unit) for the high risk neonatal population which is often challenged by severe comorbidity as well as long surgery and complicated hospital stays (NECTARINE-update, British Journal of Anaesthesia, in press).

\section{The role of research and international networking}

Paediatric anaesthesia research has for decades been neglected by national and international funding. A subsequent lack of scientific evidence has led to 'gold standards' and 'standard operating protocols' based on national and international consensus agreements by extrapolating data from the adult population. If we fully wish to be able to understand the importance of each factor involved in or responsible for the development of neurological alterations related to long and complex surgery, we will need to stress the fact that paediatric patients are not small adults. We need to understand age specific pharmacodynamics/-kinetics of anaesthetic drugs just as well as the importance of pre-, peri- and long term postoperative effects of medical supportive therapy and psychosocial patient centered care. This can only be achieved when key opinion leaders in the paediatric anaesthesia community persuade organisations, beneficiaries and politicians to invest in the future of safe paediatric anaesthesia care. We need to align our research and standard operating protocols on an international level to substantiate and align common outcome measures. Teaching, training, education and supervision of the safe conduct of anaesthesia are fundamental to continuously improve the outcome for our smallest patients.

\section{Conclusions}

The paediatric anaesthesia community is required to continuously stress the importance of the 'who', 'where', 'what', 'when' and 'how' in order to emphasize well known risk factors and causes for anesthetic morbidity. Introducing and implementing national regulations on teaching, training, education and supervision for the safe conduct of paediatric anaesthesia will result in improved perioperative outcome for our smallest patients. However, this can only be achieved if we 'speak the same language' and for this to happen we need to unite our research strategies and outcome measures.

\section{AUTHOR CONTRIBUTIONS}

All authors have equally contributed to conception, design, drafting, revision as well as acquisition, analysis and interpre- tation of data.

\section{ACKNOWLEDGMENT}

The corresponding author acknowledges the Montreal Children's Foundation for the financial support of his fellowship education in pediatric anesthesia.

\section{CONFLICT OF INTEREST}

None of the authors have any conflict of interest.

\section{REFERENCES}

[1] Weiss M, Vutskits L, Hansen TG, Engelhardt T. Safe anesthesia for every tot - the SAFETOTS initiative. Current Opinion in Anaesthesiology. 2015; 28: 302-307.

[2] Vutskits L, Culley DJ. GAS, PANDA, and MASK: no evidence of clinical anesthetic neurotoxicity! Anesthesiology. 2019; 131: 762-764.

[3] Morray JP, Geiduschek JM, Caplan RA, Posner KL, Gild WM, Cheney FW. A comparison of pediatric and adult anesthesia closed malpractice claims. Anesthesiology. 1993; 78: 461-467.

[4] Raviraj D, Engelhardt T, Giedsing Hansen T. Safe anesthesia for neonates, infants and children. Minerva Pediatrica. 2018; 70: 458-466.

[5] Jevtovic-Todorovic V, Hartman RE, Izumi Y, Benshoff ND, Dikranian K, Zorumski CF, et al. Early exposure to common anesthetic agents causes widespread neurodegeneration in the developing rat brain and persistent learning deficits. The Journal of Neuroscience. 2003; 23: 876-882.

[6] FDA. FDA drug safety communication: FDA review results in new warnings about using general anesthetics and sedation drugs in young children and pregnant women. 2016. Available at: https://www. fda. gov/Drugs/DrugSafety/ucm532356.htm (Accessed: 05 December 2020).

[7] FDA. FDA drug safety communication: FDA approves label changes for use of general anesthetic and sedation drugs in young children. 2017. Available at: https : / /www . fda.gov/downloads/Drugs / DrugSafety/UCM554644.pdf (Accessed: 05 December 2020).

[8] Jimenez N, Posner KL, Cheney FW, Caplan RA, Lee LA, Domino KB. An update on pediatric anesthesia liability: a closed claims analysis. Anesthesia \& Analgesia. 2007; 104: 147-153.

[9] Bhananker SM, Ramamoorthy C, Geiduschek JM, Posner KL, Domino KB, Haberkern CM, et al. Anesthesia-related cardiac arrest in children: update from the pediatric perioperative cardiac arrest registry. Anesthesia and Analgesia. 2007; 105: 344-350.

[10] Goyal A, Lu VM, Yolcu YU, Elminawy M, Daniels DJ. Endoscopic versus open approach in craniosynostosis repair: a systematic review and meta-analysis of perioperative outcomes. Child's Nervous System. 2018; 34: $1627-1637$

[11] Lee HQ, Hutson JM, Wray AC, Lo PA, Chong DK, Holmes AD, et al. Analysis of morbidity and mortality in surgical management of craniosynostosis. Journal of Craniofacial Surgery. 2012; 23: 1256-1261.

[12] Lal DR, Gadepalli SK, Downard CD, Ostlie DJ, Minneci PC, Swedler $\mathrm{RM}$, et al. Perioperative management and outcomes of esophageal atresia and tracheoesophageal fistula. Journal of Pediatric Surgery. 2017; 52: $1245-1251$

[13] Oomen MW. Systematic review of the literature: comparison of open and minimal access surgery (thoracoscopic repair) of esophageal atresia with tracheo-esophageal fistula (EA-TEF). In Nazari S (ed.) Front Lines of Thoracic Surgery (pp. 309-320). IntechOpen. 2012.

[14] Leeuwen L, Fitzgerald DA. Congenital diaphragmatic hernia. Journal of Paediatrics and Child Health. 2014; 50: 667-673.

[15] Dingeldein M. Congenital diaphragmatic hernia: management \& outcomes. Advances in Pediatrics. 2018; 65: 241-247.

[16] Wang Y, Honeyford K, Aylin P, Bottle A, Giuliani S. One-year outcomes for congenital diaphragmatic hernia. BJS Open. 2019; 3: 305-313.

[17] Brown KL, Pagel C, Ridout D, Wray J, Anderson D, Barron DJ, et al. 
What are the important morbidities associated with paediatric cardiac surgery? A mixed methods study. BMJ Open. 2019; 9: e028533.

[18] Green MD, Parker DM, Everett AD, Vricella L, Jacobs ML, Jacobs JP, et al. Cardiac biomarkers associated with hospital length of stay after pediatric congenital heart surgery. The Annals of Thoracic Surgery. 2020: S0003-4975(20)31354-0

[19] Liu M, Druschel CM, Hannan EL. Risk-adjusted prolonged length of stay as an alternative outcome measure for pediatric congenital cardiac surgery. The Annals of Thoracic Surgery. 2014; 97: 2154-2159.

[20] Spector LG, Menk JS, Knight JH, McCracken C, Thomas AS, Vinocur $\mathrm{JM}$, et al. Trends in long-term mortality after congenital heart surgery. Journal of the American College of Cardiology. 2018; 71: 2434-2446.

[21] Tai D, Dick P, To T, Wright JG. Development of pediatric comorbidity prediction model. Archives of Pediatrics \& Adolescent Medicine. 2006; 160: 293-299.

[22] Stolwijk LJ, Lemmers PM, Harmsen M, Groenendaal F, de Vries LS, van der Zee DC, et al. Neurodevelopmental outcomes after neonatal surgery for major noncardiac anomalies. Pediatrics. 2016; 137: e20151728.

[23] Disma N, Leva B, Dowell J, Veyckemans F, Habre W. Assessing anaesthesia practice in the vulnerable age group: NECTARINE: a European prospective multicentre observational study. European Journal of Anaesthesiology. 2016; 33: 233-235.

[24] Auroy Y, Ecoffey C, Messiah A, Rouvier B. Relationship between complications of pediatric anesthesia and volume of pediatric anesthetics. Anesthesia and Analgesia. 1997; 84: 234-235.

[25] NCEPOD. National confidential enquiries into perioperative death. 1993. Available at: http://www.ncepod.org.uk (Accessed: 05 December 2020).

[26] Harrison TE. Engelhardt T. McFarlane F, Flick RP. Regionalization of pediatric anaesthesia care: has the time come? Paediatric Anaesthesia. 2014; $24: 897-898$.

[27] Danish Health Authorities. Recommendations from the Danish Health Authority. Thesis Guidance Anaethesiology. 2020. Available at: https://www.sst.dk/-/media/Viden/Specialplaner/ Specialeplan-for-anæstesiologi/SST_Specialevejledning_ for_Anaestesiologi-03072020.ashx?la=da\&hash= 6B3BB5148B1CDOF7AE9035038D3AA05E08997EDA (Accessed: 05 December 2020).

[28] ADARPEF. Recommendations from L'association des Anesthésistes Réanimateurs Pédiatriques d'Expression Française (ADARPEF) / Société Française d'Anesthésie et de Réanimation (SFAR). 2004. Available at: http://e-adarpef.fr/publications/mises-au-point/ sros-et-recommandations/ (Accessed: 05 December 2020).

[29] Habre W, Disma N, Virag K, Becke K, Hansen TG, Jöhr M, et al. Incidence of severe critical events in paediatric anaesthesia (APRICOT): a prospective multicentre observational study in 261 hospitals in Europe. The Lancet Respiratory Medicine. 2017; 5: 412-425.

[30] Stricker PA, de Graaff JC, Vutskits L, Muhly WT, Xu T, Torborg AM, et al. Pediatric perioperative outcomes group: defining core outcomes for pediatric anesthesia and perioperative medicine. Pediatric Anesthesia. 2018; $28: 314-315$.

How to cite this article: Mathias Johansen, Vincent Collard, Thomas Engelhardt. Why complex surgeries in children are associated with poorer outcomes. Signa Vitae. 2021;17(3):5-9. doi:10.22514/sv.2021.060. 